\title{
Pseudoachondroplasia: A Rare Cause of Short Limbed Dwarfism
}

\author{
K Jagadish Kumar, ${ }^{1, *}$ Nayana, ${ }^{1}$ V G Manjunath, ${ }^{1}$ and Chandrashekar Shetty ${ }^{1}$ \\ ${ }^{1}$ JSS Medical College, Mysore, India \\ "Corresponding author: K Jagadish Kumar, JSS Medical College, Mysore, India. E-mail: jagdishmandya@gmail.com
}

Received 2016 June 14; Revised 2017 February 04; Accepted 2017 February 07.

\begin{abstract}
Pseudoachondroplasia is a rare type of short-limbed skeletal dysplasia. It is usually found as an autosomal dominant inheritable disorder. Children are normal at birth and they present developmental delay in walking by the age of 2, an abnormal waddling gait or deformities of the lower limb. Diagnosis is based on characteristic clinical and radiological findings. This study reports on a 6-year-old boy with classical features of pseudoachondroplasia.
\end{abstract}

Keywords: Pseudoachondroplasia, Short Limbed Dwarf, Skeletal Survey

\section{Introduction}

Pseudoachondroplasia (PSACH) is a rare type of shortlimbed dwarfism. The prevalence of Pseudoachondroplasia is approximately 4 per 1 million individuals (1). It is usually found as an autosomal dominant disorder, yet somatic or germline mosaicism has also been reported. Children are normal at birth and they usually present short stature, a delay in walking, an abnormal waddling gait or lower limb deformity at 2 to 3 years of age. Radiologically both epiphysis and metaphysis will be affected.

\section{Case Presentation}

A 6-year-old child born of a consanguineous marriage was referred to our hospital for evaluation of short stature. According to the parents, the boy was normal during the first 2 years of life, after which they noticed a short stature and abnormal waddling gait. On examination, the child had height of $84 \mathrm{~cm}\left(<3^{\text {rd }}\right.$ percentile $)$ and upper to lower segment ratio was 1.64:1 suggesting short limbed dwarfism. The child had wrist widening, short stubby fingers, lumbar lordosis and genu varus deformity. The face appeared normal (Figure 1). Systemic examination and intelligent quotient was normal. None of the family members were dwarfs. Skeletal survey was done in view of the short stature.

Radiographic findings included a normal skull, with chest X-ray dipicting spatulate ribs with anterior widening. Wrist X-ray revealed delay in bone age with epiphyseal end of bones showing sclerosis, widening, and irregularities. Epiphysis of radius and carpal were less dense and had pencil line outline for expected age. Pelvis showed short thickened femur and tibia, widening of the metaphysis of
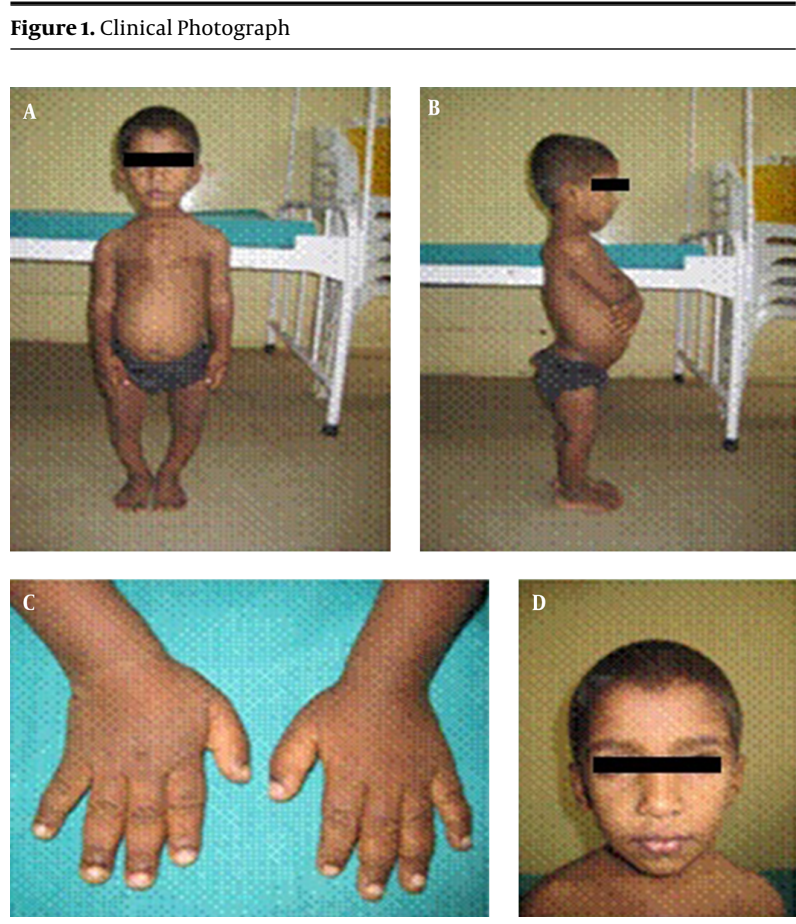

A, genu varus; B, lumbar lordosis; C, short stubby fingers; D, normal face.

both of these bones and poorly formed acetabulam. Lumbosacral spine revealed normal interpedicular distance, platyspondyly, superior and inferior rounding of vertebral bodies (Figure 2). Serum calcium, phosphorous, alkaline phosphatase, blood urea, and creatinine were within normal limits. Ultrasound of the abdomen was normal. Based on clinical and radiological features, a diagnosis of pseudoachondroplasia was made. 
Figure 2. Radiological Findings
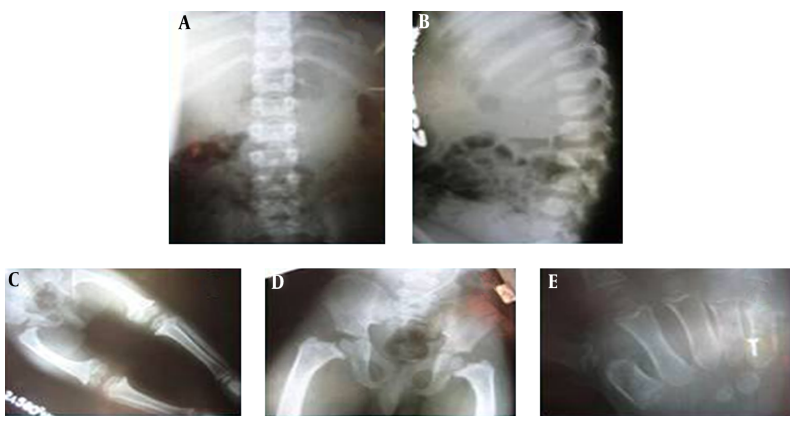

A, normal interpedicular distance; B, superior and inferior rounding of vertebral bodies; C, short thickened femur and tibia with widening of the metaphysis; $\mathrm{D}$, poorly formed acetabulam; E, pencil line outline for expected age.

\section{Discussion}

Pseudoachondroplasia is an uncommon type of skeletal dysplasia with short limbs, which occurs secondarily to mutations within genes encoding cartilage oligomeric matrix protein (COMP) on chromosome 19. This COMP is present in the extracellular matrix of the ligaments, tendons, and cartilages (1). The disorder was first described by Maroteaux and Lamy in 1959. It is referred as "Achondroplasia with a normal face" (2).

In most families, it runs an autosomal dominant inheritance pattern, however, in some cases, germline or somatic mutations have been reported (3). In our case, the parents and younger siblings were normal, thus indicating the probability of germline or somatic mosaicism in one of the parents. Children are usually invariably normal at birth, and they show a developmental delay in walking, an abnormal waddling gait or deformities of the lower limbs by 2 years of age (4). The case presented here was also normal upto 2 years of age, then presented a waddling gait. In pseudoachondroplasia, length is normal at birth (4). On physical examination, they have normal faces and normal intelligent quotient (5). The adult height usually achieves between 82 and $130 \mathrm{~cm}$ with gross shortening of the limbs. Other associated deformities include genu valgum, genu varum, genu recurvatum, elbow extension limitation, kyphoscoliosis, lumbar lordosis, and joint laxity with secondary osteoarthritic changes (1).

The case of the current report also had lumbar lordosis (Figure 1). Lumbar lordosis is observed in around 50\% of affected individuals (4). Shortening of the long bones, predominantly of femur and humerus with flared irregular metaphysis are important characteristic findings (5). The radiographic features include, a normal skull, spatulate ribs with anterior widening, pelvis with poorly formed acetabulam with rounded iliac wings. Spine X-ray shows platyspondyly, anterior tongue like projection, superior and inferior rounding of vertebral bodies and normal interpedicular distance. Limb X-ray reveals short thickened femur and tibia with metaphyseal widening.

Ossification of epiphysis is delayed, and when they ossify they are irregular, small, and fragmented. The commonly affected epiphysis are the femoral capital and humeral epiphysis. In the femur bone, medial beaking of the femoral neck is a characteristic finding $(5,6)$. Hand and foot bones are short, broad with small and rudimentary epiphysis (2). The skull and facial bones are normal (5). The diagnosis of pseudoachondroplasia is based on clinical presentation, examintionl, and radiological findings (4).

The differential diagnoses of pseudoachondroplasia are achondroplasia, multiple epiphyseal dysplasia (MED), and congenital spondyloepiphyseal dysplasia (SED) (4).

Achondroplasia children have a large head with prominent frontal bones and a narrow base. In the lumbar region, the interpedicular distance decreases caudally, yet, with normal vertebral height. Only metaphysis is effected (4). In MED, epiphyses are abnormal with a normal metaphysis, pelvis, and spine. Even though waddling gait is present, yet, is less consistent when compared to pseudoachondroplasia. The spine is usually normal $(4,5)$. In Spondyloepiphyseal Dysplasia, congenital epiphyseal changes mimic pseudoachondroplasia, yet, spinal changes are more pronounced with marked kyphoscoliosis. Hip joints are affected disproportionately when compared to nearly normal distal limbs (5-7).

Skeletal complications are common in children with Pseudoachondroplasia because of early degeneration of the joints. Therefore, clinicians should diagnose the condition for the purpose of genetic counseling, and early theatment of joint manifestations (5). Many patients have early osteoarthritic changes and joint deformities, which requires orthopedic intervention. At the genetic level, COMP altering therapy can be used to treat this condition (5).

\subsection{Conclusion}

Pseudoachondroplasia is an uncommon form of short limbed skeletal dysplasia wherein the patients usually have skeletal complications because of early degeneration of the joints.

Early recognition and diagnosis is necessary for genetic counseling and early treatment of joint manifestations. 


\section{References}

1. Herring JA, Tachdjian MO. Pediatric orthopedics. 4th ed. 2. Philadelphia: Saunders; 2008. pp. 1702-7.

2. Lachman RS. Skeletal dysplasias. 2008 :2613-70. doi: 10.1016/b978-0-32304520-9.50173-8.

3. Khungar A, Mahajan P, Gupte G, Vasundhara M, Kher A, Bharucha BA. Pseudoachondroplastic dysplasia. J Postgrad Med. 1993;39(2):91-3. [PubMed: 8169872].
4. Briggs MD, Wright MJ. Pseudoachondroplasia University of Washington, Seattle; 2004. [updated 2015].

5. Tandon A, Bhargava SK, Goel S, Bhatt S. Pseudoachondroplasia: A rare cause of rhizomelic dwarfism. Indian J Orthop. 2008;42(4):477-9. doi: 10.4103/0019-5413.43400. [PubMed: 19753240].

6. Radlovic V, Smoljanic Z, Radlovic N, Jakovljevic M, Lekovic Z, Ducic S, et al. Pseudoachondroplasia: A case report. Srp Arh Celok Lek. 2013;141(910):676-9. doi: 10.2298/sarh1310676r.

7. Douglas PB, Robert LE, Justin QL, Thomas P. Pseudoachondroplasia. Appl Radiol Online. 2006;35(7). 